\title{
Time and spatial resolution of slip and twinning in a grain embedded within a magnesium polycrystal
}

\author{
P. A. Lynch ${ }^{1}$, M. Kunz ${ }^{2}$, N. Tamura ${ }^{2}$, M. R. Barnett ${ }^{1}$
}

1. ARC Centre of Excellence for Desing in Light Metals, Institute for Frontier Materials. Deakin University. 75 Pigdons Road, Waurn Ponds, Victoria, 3216. Australia. email: Peter.Lynch@deakin.edu.au._Phone: + 61352272641

2. Advanced Light Source, Lawrence Berkeley National Laboratory, 1 Cyclotron Road, Berkeley, California 94720. U. S. A. email: ntamura@1bl.gov. Phone: +1 510486 6189

\begin{abstract}
Plastic yielding in magnesium alloys frequently involves the initiation of both slip and twinning events. Proper understanding of the phenomenon at the grain level requires knowledge of how these two mechanisms progress and interact over both time and space and what the local resolved stresses are. To date, simultaneous collection of such information has been out of reach. To address this shortfall, we have developed a modified Laue based in-situ micro X-ray diffraction technique with unprecedented combination of time and spatial resolution. A ten-fold reduction in data collection times is realised by refinement of rapid polychromatic Laue 'single-shot' mapping. From single Laue patterns, we extract grain depth information, detect onset of yielding and achieve $2 \times 10^{-4}$ lattice strain resolution. The technique is employed to examine yielding and twinning in a magnesium grain embedded $\sim 200 \mu \mathrm{m}$ below the sample surface. We examine 13 time steps and reveal the following behaviour: initial onset of basal slip, subsequent onset of twinning, development of further accommodation slip and evolution of twin shape and size; along with the corresponding values of local resolved shear stresses.
\end{abstract}

(C) 2014. This manuscript version is made available under the Elsevier user license http://www.elsevier.com/open-access/userlicense/1.0/ 


\section{Introduction}

Plastic yielding in magnesium alloys frequently involves the initiation of both slip and twinning events. Proper understanding of the phenomenon at the grain level requires knowledge of how these two mechanisms progress over both time and space and what the local stresses are [1,2]. To date, experimental identification of active deformation mechanisms along with simultaneous monitoring of their variation over time and space has proved challenging. To address this shortfall, we develop herein a modified Laue in-situ micro X-ray diffraction technique with the required combination of time and spatial resolution. The technique is employed to determine the onset and progress of slip and twinning in a magnesium grain embedded $\sim 200 \mu \mathrm{m}$ below the sample surface.

In-situ neutron diffraction has been widely employed to probe the average response of similarly diffracting grains in metals yielding by twinning [3-8]. Magnesium has become somewhat of a model hcp material for such study since Ghargouri et al. [8] showed how changes in the diffracted intensity reveal the onset of deformation twinning and used this to demonstrate that $\{10 \overline{1} 2\}<\overline{1} 011>$ twinning in magnesium follows a critical resolved shear stress law. This agrees well with the theoretical treatment by Lebensohn and Tome [9] and is an important point for the present study because resolved shear stresses can be adequately obtained from the deviatoric strains that are provided by Laue type techniques. Neutron diffraction has also been combined with crystal plasticity by a number of workers to extract effective mean critical resolved shear stresses for slip and twinning modes $[4,7,10]$. The stresses for the onset of twinning were found to exceed those for basal slip by a factor of 2-7 times. It is therefore quite likely that for a wide class of grain orientations, 'micro' yielding due to basal slip will precede twinning. This is likely to impact significantly on subsequent twinning events via a number of mechanisms; for example, by stimulating nucleation [11], by facilitating initial growth [12-14], and ultimately by impeding growth [15, 16]. Bulk neutron methods have also shown that newly formed twins display relaxed [3] and even reversed [7] internal stresses. The magnitude of these stresses will control the activation of subsequent slip modes but because of their transient nature, diffraction data averaged over many grains is not able to reveal the sequence of deformation with necessary precision.

Steps towards addressing this problem have been taken by Aydiner et al. [17], who employed a high-energy synchrotron experiment to isolate radiation diffracting from a single embedded grain in a deforming magnesium AZ31 alloy. Seven time steps - levels of imposed stress - 
were examined and full stress tensors were obtained at the grain level. Two twins were seen to form at a resolved stress somewhere between $\sim 22$ and $42 \mathrm{MPa}$, a range set by the chosen loading increment. Resolved shear stresses in the twins were seen to be relaxed compared to the parent and ranged between -10 MPa and $14 \mathrm{MPa}$. But no information was obtained on any prior, concurrent, or subsequent, basal slip, nor is spatial intragranular information available with this technique. To access intragranular information, Balogh et al. [18] applied differential aperture x-ray diffraction (DAXM) [19] to study an embedded twinned grain under load in a magnesium AZ31 alloy. The technique is attractive because it provides unprecedented spatial resolution in three dimensions. Analysis was restricted to a single component of the stress tensor and this revealed significant gradients over the grain and in the twin domain. However, the development of the stresses and structure with increasing stress is not practically accessible using DAXM due to multiple scans required at each time (stress) step.

So it is seen that while considerable progress has been made, to date it has not been possible to simultaneously correlate spatial and time resolution to the extent desired to observe the variation of slip and twinning events in time and with increasing stress during the yielding of magnesium. The present study addresses this problem and reports observations of the progress of slip and twinning in an embedded magnesium grain during tensile testing. The paper is organized as follows: first the modified Laue technique developed for the study is described in some detail, then findings are presented in terms of measured diffraction intensities, peak broadening and micro-strains. The paper concludes with our interpretation of how slip and twinning interact in the present material.

\section{Experimental methodology}

The modified Laue technique employed for this study is based on a tensile test experiment carried out on the synchrotron micro-XRD beamline at the Advanced Light Source [20] operating in polychromatic mode with a spatial resolution of $0.8 \mu \mathrm{m}^{2}$. Short exposure times and real-time detector readout ensures a dwell time of less than a second. Spatial resolution is obtained via rapid scanning in two dimensions in which pixels $0.8 \mu \mathrm{m}^{2}$ by the grain depths define the diffracting volumes (long columns) at each point.

The experiment was performed using a reflection geometry as shown in Figure 1a. The incident beam sample angle $(\omega)$ was set at $\omega=30^{\circ}$ with the X-ray detector positioned $140 \mathrm{~mm}$ away from the sample at an angle of $2 \theta=90^{\circ}$. Prior to loading, and at each load step, the 
sample surface is precisely aligned using a laser triangulation system, such that the focal plane of the incident beam is coplanar with the $\mathrm{X} / \mathrm{Y}$ axes of the raster scan. With the simultaneous grain tracking used during loading, for each load increment the same raster scan stage settings could be repeated. Sample tracking was achieved using a 'real time' fiducial marker feedback loop as described in [21].

Laue diffraction patterns were collected on a large area Pilatus detector and their crystallographic indexation was carried out using the micro-XRD software 'X-ray Microdiffraction Analysis Software' (XMAS) [22]. The indexing procedure provides the crystallographic grain orientation and the deviatoric lattice strains. As indicated in Figure 1b, Laue patterns from multiple grains (up to six in the present study) contribute to the collected pattern. Each grain has a different diffracting centre ('point of origin'). The conventional automated approach [23] is not able to identify and index individual patterns with the required precision. To address this, two modifications were made to the technique: i) a new Laue pattern peak profile fitting protocol was developed with the aim of achieving a 0.1 pixel accuracy in the peak location across the entire $60 \mathrm{MPa}$ loading range, even when moderate levels of plastic deformation are present, and ii) a new 'single-shot' approach was developed to measure and correct for the different points of origin of the diffracting grains.

Accurate identification of the peak position was achieved by fitting the detected Laue spots with 2D functions within a scalable box window [24]. The approach helps to overcome problems introduced by the deterioration of Laue reflections with plastic deformation (either during the test or due to sample preparation), the development of peak overlap and the relative change in intensity of the individual $h k l$ spot in a single Laue pattern. The scalable box window technique consistently identifies the most intense part of a distorted Laue spot. Careful inspection was also employed to identify the particular $h k l$ reflections ( 27 of them for the parent grain) that would be employed for indexation throughout the test. Thus diffraction peaks that fell near detector edges or peaks that developed substantial overlap during the deformation were excluded.

The material examined was a commercial extruded magnesium alloy AZ31 (nominally 3\% Al and $1 \% \mathrm{Zn}$ by weight and grain size of $100 \mu \mathrm{m})$. A tensile sample was cut from across the face of the extruded bar such that its long (loading) axis was perpendicular to the original extrusion axis. After standard metallographic preparation, an electron backscatter diffraction (EBSD) grain orientation map was recorded over a $400 \mu \mathrm{m} \times 400 \mu \mathrm{m}$ region in the middle of 
the tensile gauge length. This served two purposes, the surface grain orientation results were used to verify the Micro-XRD data and the surface grain information provided an independent measure to test the single shot approach for depth recovery. Micro-XRD data was collected across the same region of interest as identified for the EBSD measurements in a raster step size of $12 \mu \mathrm{m}(\mathrm{x}) \mathrm{x} 20 \mu \mathrm{m}(\mathrm{y})$. This can be tailored as required and these coarse settings were chosen in view of the coarse grain size. The time taken to scan this area was less than 8 minutes.

In the present experiment the stress is increased over time and so the key interest is in how deformation evolves with increasing stress. The experiment thus involves interruptions at constant stress values to collect data. 'Fine' loading increments of $5 \mathrm{MPa}$ were employed. In total 13 load increments were examined up to an applied stress of $70 \mathrm{MPa}$. In total, data for 34 grains was captured, however, only a single grain is examined here due to the insight it provides on the slip-twinning interactions at the intragranular level. The orientation of the selected grain is shown in the basal pole figure generated using the Micro-XRD in Figure 2a. The c-axis of the grain is inclined at $35^{\circ}$ with respect to the loading direction, the maximum Schmid factor for $\{10 \overline{1} 2\}<\overline{1} 011>$ twinning is 0.33 and that for basal slip is 0.46 .

Prior to loading the sample, detector triangulation maps were recorded according to the method described in [25] to determine the depth of each grain below the sample surface. A reconstructed image of the selected grain, showing the Euler $\theta$ angle (defined in Bunge convention: $\varphi 1: 123.74, \Phi: 79.21, \varphi 2:-154)$ of the chosen grain is given in Figure $2 \mathrm{~b}$. The grain displays a narrow orientation spread of $0.06^{\circ}$ which confirms the strain free starting condition. It is a large grain $180 \mu \mathrm{m}$ in diameter located approximately $200 \mu \mathrm{m}$ below the sample surface. As will be described in detail below, a $\{10 \overline{1} 2\}<\overline{1} 011>$ twin was observed to form within this grain and the location of the twin is indicated in Figure 2c.

Returning now to the requirement to determine the location of the 'point of origin' of the diffracting volume, to determine this, we note that the grain depth impacts most significantly on the magnitude of the $\varepsilon_{\mathrm{yz}}$ strain term determined during the indexing procedure. This can be verified theoretically [26] but here we provide experimental evidence using a single crystal stage translation experiment and show how this term can be employed to provide a rapid depth estimate. 
First, a single crystal (silicon) surface was aligned with the laser triangulation system to establish a 'zero' position. Using a conventional scattering geometry $\left(\omega=45^{\circ}\right.$ and $\left.2 \theta=90^{\circ}\right)$ Laue patterns were collected while translating the stage in the $\mathrm{z}$ direction in $10 \mu \mathrm{m}$ increments. To mimic the effect expected for an embedded grain, crystallographic indexation and subsequent deviatoric strain determination was then carried out using the original experimental 'zero' calibration settings. The deviatoric strains are plotted in Figure 3a against $\mathrm{z}$-translation position. It is clear that the $\mathrm{z}$-shift alters the calculated strain, particularly the $\varepsilon_{\mathrm{yz}}$ shear strain component. We propose that the relationship between this component and the ztranslation provides an effective calibration curve for determining the depth of the "point of origin' of a diffraction pattern emanating from a location beneath the sample surface. The basis of this procedure implies that the sample exhibits a relatively strain free starting condition, free from plastic deformation. A result confirmed by the recorded Laue patterns at an imposed load of $12 \mathrm{MPa}$ and from bulk X-ray diffraction residual strain measurements carried out on the sample prior to loading.

To test this approach a nominal load $(12 \mathrm{MPa})$ was applied to the present sample, to eliminate any slack in the system and the measured $\varepsilon_{\mathrm{yz}}$ strain terms were then 'zeroed' by adjusting the point of origin along the $\mathrm{z}$ axis. The established depths (in the form of the YCent value [22]) are then maintained for the remainder of the test. Figure $3 \mathrm{~b}$ shows a comparison of the grain depth locations obtained using this technique with those obtained using detector triangulation. The data in the figure are for a single row (for $\mathrm{y}=-5932 \mu \mathrm{m}$ ) of grain depth information for an arbitrary grain. There is good agreement between the two measures, as illustrated both methods exhibit the same grain depth (morphology) features with $\mathrm{x}$-translation across the grain. Overall the magnitude of the depth data sets appear reasonable based on a triangulation uncertainty of about 20 micron ( $~ 0.1$ detector pixel resolution) coupled with the potential uncertainty in the detector translation reproducibility. The triangulation depth result at the Xstage position of $10044 \mu \mathrm{m}$ does appear excessive and does not conform to the grain depth morphology. This single result is considered as an experimental outlier, potentially caused by mechanical hardware instability during data collection. The overall agreement between the two independent data sets suggests that the 'single shot' methodology is considerably more time efficient than a full triangulation experiment since collection of data over several detector positions is avoided. 


\section{Results}

In the following section we present micro-diffraction data in terms of grain orientation, diffraction peak intensities, diffraction peak streaking/broadening and measured microstrains.

\section{Orientation and diffraction peak intensity - identifying twin formation}

The onset of yielding by twinning is revealed in the changes seen in the intensities of individual Laue reflections. For precise measurement of the integrated intensity, by application of the peak fitting protocol developed, a global box size was defined for each Bragg reflection across the entire loading range (from 12 to $68 \mathrm{MPa}$ ). This avoids the potential of an integrated intensity decrease caused by plastic deformation induced peak broadening beyond the box perimeter. The mean integrated intensity from the $(21 \overline{3} 0)$ reflection across the parent grain is plotted against imposed stress in Figure 4. A drop in intensity can be seen to begin at imposed stress levels of between 54 and $58 \mathrm{MPa}$. The change is abrupt and is a hallmark of the reorientation that accompanies twinning. In a subsequent stress increment it was possible to identify reflections from an orientation re-oriented according to the $\{10 \overline{1} 2\}<\overline{1} 011>$ twin relation [27]. The active twin variant was identified to be $(0 \overline{1} 12)[01 \overline{1} 1]$ with a Schmid factor of 0.33 (compared to $0.21,0.21,0.26,0.32,0.16$ for the other systems). Thus we see that the twin system with the highest Schmid factor is active. The intensities of the $(\overline{2} 114)$ reflections from the twinned orientation are also included in Figure 4. Evidently, in the present experimental set up, the drop in parent intensity is a more sensitive measure to the onset of twinning than the appearance of an 'indexible' set of daughter reflections.

The spatial distributions of the parent $(21 \overline{3} 0)$ and the twin $(\overline{2} 114)$ reflections are plotted in Figure 5 as a function of applied stress. The images are presented against $\mathrm{x}-\mathrm{y}$ positions and it can be seen that the grain tracking methodology works well [21]. It can also be seen that as soon as the twin is evident, at an applied stress of $54 \mathrm{MPa}$ (Figure 5b), it adopts an ellipsoidal shape and spans the parent grain. This is consistent with an initial burst of rapid twin growth over the parent cross section [1]. The trace of the $\mathrm{K}_{1}(01 \overline{1} 2)$ twinning plane is superimposed on the $\mathrm{x}-\mathrm{y}$ raster scan in Figure $5 \mathrm{~b}$ (dashed line) and it is seen that the elongation of the twin projection follows this trace. This is support for the coincidence of the twin habit and $\mathrm{K}_{1}$ 
planes. The habit plane is represented above in Figure $2 \mathrm{c}$ as a grey plane given in terms of the sample (dashed line) XYZ reference frame.

With increasing levels of imposed stress, the twin is seen to thicken, as witnessed by the increase in both its integrated intensity and the area it occupies in the $x-y$ mapping plane (Figure 5). At the midpoint of the twin the parent intensity is $78 \%$ of its previous untwinned value of $1.15 \times 10^{6}$ counts. The grain thickness at this point is $160 \mu \mathrm{m}$ parallel to the incoming beam. So the maximum twin thickness in this direction is $0.22 \times 160=35 \mu \mathrm{m}$. The incoming beam makes an angle of $74.4^{\circ}$ with the twin plane normal, which means the twin minor axis thickness is $9.4 \mu \mathrm{m}$ at $64 \mathrm{MPa}$, increasing to $11.3 \mu \mathrm{m}$ at $68 \mathrm{MPa}$. Where, the parent grain midpoint intensities are $0.90 \times 10^{6}$ and $0.87 \times 10^{6}$ at $64 \mathrm{MPa}$ and $68 \mathrm{MPa}$ respectively.

\section{Orientation and mosaic spread - yielding by dislocation glide}

In addition to providing information on the onset of twinning, the present technique also reveals the earlier onset of dislocation activity. The crystal orientation determined using the present approach is particularly sensitive to slip induced lattice rotation (the orientation precision can approach $0.001^{\circ}$, based on all Laue reflections of a grain [28]. The Laue diffraction spots also are highly sensitive to the 'mosaic' spread (pattern streaking) that arises with the accumulation of geometrically necessary dislocations and boundaries [26].

The lattice orientation for a single point within the parent grain is plotted against applied stress in Figure 6a in terms of the misorientation between it and its orientation at the beginning of the deformation. A very slight increase in misorientation accompanies the stress in the elastic region. A change in the rate of grain rotation can be detected at an applied stress level of between 43 and $46 \mathrm{MPa}$. This provides circumstantial evidence for the onset of plasticity, which inevitably causes some degree of lattice rotation. Further evidence for dislocation mediated plasticity is provided by the full width half maximum (FWHM) values of the $(21 \overline{3} 0)$ parent and $(\overline{2} 114)$ twin reflections plotted in Figure $6 \mathrm{~b}$. These values are averaged over the grain so are a little coarser, than the data in Figure 6a. In this case an increase in the FWHM can be seen to occur at an applied stress of between 51 and $54 \mathrm{MPa}$. Evidently sufficient dislocation activity has built up by these stress levels to create the detected lattice spread. Recall from the above that twin onset was not seen to occur until slightly later in the deformation (applied stresses of between 54 and $58 \mathrm{MPa}$ ). 
The spatial distribution of the mosaic spread (FWHM) over the grain of interest before and after twinning is shown in Figure 7. Before twinning (Figure 7a), the FWHM values vary randomly and slightly over the parent grain. However, following the appearance of the twin (Figure $7 \mathrm{~b}$ ), there is a significant increase in the FWHM values, from $\sim 0.6^{\circ}$ to $\sim 4.0^{\circ}$. It is also clear that regions of high mosaic spread are concentrated at the ends of the twin, indicative of dislocation glide that has initiated to accommodate the stress field of the twin.

Due to the ease of basal slip in magnesium [29-31], it is highly likely that the measured mosaic spread is dominated by this glide mode. Using the method presented by Barabash et al. [32] and applied, for example, to CP-Ti by Bieler et al [33], Laue pattern streaking was simulated based on the potential slip systems for the $h c p$ case. Simulated streak behaviour and the corresponding experimental data from five reflections taken from a single Laue pattern collected at $64 \mathrm{MPa}$ are shown in Figure 8. The simulated patterns based on the (0002)[1120] slip system match well with the observations. This is consistent with our hypothesis. However, it should be noted that $<\mathrm{c}+\mathrm{a}>$ pyramidal slip gives similar predictions so this mode cannot be categorically discounted. The dominance of basal slip is also supported by the local stress data that will be presented further below.

From the measured peak spread $\left(3.6^{\circ}\right)$ and with knowledge of the diffracting column length at the twin tip $(120 \mu \mathrm{m})$, the lattice curvature, $R$, across the grain at this point was determined. The Cahn-Nye relationship ( $\rho=1 / R b$ where $b$ is the Burgers vector, assumed to be $0.312 \mathrm{~nm}$ in the present case) provides a first order estimate of the geometrically necessary dislocation density needed to account for the curvature. At $64 \mathrm{MPa}$, the density is $\sim 0.17 \mathrm{x}$ $10^{9} \mathrm{~cm}^{2}$ at the location shown in Figure $7 \mathrm{~b}$, with a slight increase to $\sim 0.2 \times 10^{9} \mathrm{~cm}^{2}$ at 68 $\mathrm{MPa}$.

Turning now to the twin, apart from a single point, the mosaic spread is fairly uniform in the twin at an applied stress level of $64 \mathrm{MPa}$; ranging from 0.7 up to $1.8^{\circ}$ (not shown). Upon further loading, this range increases from 1.2 to $2.5^{\circ}$ at $68 \mathrm{MPa}$ (Figure 7). These values are lower than those in the parent but this is to be expected in part due to the smaller twin volume. As in the case of the parent grain, peak streaking is consistent with the presence of (0002)[11 $\overline{2} 0]$ basal geometrically necessary dislocations (Figure 8). However, the peaks also appear to have widened perpendicular to the streaking axis ( $v$-axis) [32]. This may be indicative of an increase in the total dislocation density, suggesting a possible enhancement of random dislocation trapping, perhaps due to 'transformed' [34] dislocations in the twin 
interiors. The calculated density of geometically necessary $<a>$ dislocations for the point shown (diffracting 'length' $=35 \mu \mathrm{m}$ ) is $\sim 0.1 \times 10^{9} \mathrm{~cm}^{2}$ at $64 \mathrm{MPa}$ and $\sim 0.13 \times 10^{9} \mathrm{~cm}^{2}$ at 68 $\mathrm{MPa}$. It thus appears that there is considerable basal dislocation activity in the twin interior, even at these early stages of formation.

\section{Resolved shear stresses}

The present Laue based technique provides deviatoric lattice strains which can be converted to deviatoric stresses, via the elastic compliance tensor, and resolved, via the Schmid tensor, to give the shear stresses on the active slip and twinning systems. This has to be done with some caution; strain accuracy is best until the onset of significant streaking of the Laue spots. The onset of twinning also impairs strain accuracy due to spatial perturbation of the diffracting volumes. Due to these effects, we focus our analysis to grain average data and to maximum levels of applied stress of $74 \mathrm{MPa}$. In this range we obtain a strain component sensitivity of $2 \times 10^{-4}$ in the deviatoric strains. Strain component sensitivity can be an issue with the present technique [30] but it is clear that acceptable levels can be obtained with the approach employed in the present study.

The distribution of the resolved stresses on the most heavily stressed basal slip system (0002) $[11 \overline{2} 0]$ in the parent grain $($ Schmid factor $=0.46)$ is shown in Figure 9 for an imposed stress of $38 \mathrm{MPa}$. Magnesium is nearly elastically isotropic so no systematic variation in stresses over the grain is expected at these low levels of applied stress and this is confirmed by the map in Figure 9a.The variation present can be ascribed to the noise inherent in the present technique. From the histogram in Figure $9 \mathrm{~b}$ we can see that the variation can be approximated with a normal distribution (mean $=15.5 \mathrm{MPa}$ and standard deviation $=3.2$ $\mathrm{MPa})$.

Similar data to that presented in Figure 9 is presented in Figure 10 for stresses resolved onto the active twinning system but this time the applied load is higher: $64 \mathrm{MPa}$. The mean value is $19.5 \mathrm{MPa}$ and the standard deviation over the grain evident in the distribution curve drawn through the data in Figure 10b is similar to that seen for the basal slip results in Figure 9b. Interestingly, in this case the stresses do not appear to be varying randomly over the grain and although the noise level is quite high, there appears to be a relaxation of stresses adjacent to the twin and along the top and far left of the parent grain. The first of these observations is according to expectation that a twin should relax the stresses in its vicinity. Certainly, it is 
quite clear in Figure 10a that the stresses in the twin are considerably lower than those in the parent, showing relaxation of internal stresses in the twin domain.

The values of the resolved stresses averaged over the grain and twin are plotted against the applied stress in Figure 11. Up until an imposed stress of $43 \mathrm{MPa}$ the experimental values fall within $3 \mathrm{MPa}$ of the predicted stresses. At higher loadings, deviations are seen from elastic predictions due to the onset of plasticity in the grain of interest and probably due also to plasticity elsewhere in the sample. The plateau attained in the shear stresses on the most heavily stressed basal plane is strong evidence that this slip system is active (e.g. [35]). The mean stresses on the active twinning system show less of a systematic trend but it is apparent that in the two loading steps prior to the appearance of the twin reflections, the twinning system accumulated stresses at an increased rate. Once the twin reflections are seen, the mean stresses relax by approximately $6 \mathrm{MPa}$. The average stresses on the active twinning system in the twin (i.e. the shear stress on the same plane and direction in the sample co-ordinates as in the parent) are seen to be significantly less than in the parent prior to twin inception by approximately $20 \mathrm{MPa}$. However, the average stresses on the most heavily stressed basal system in the twin, which is on a different plane in the sample co-ordinates to that in the parent, are less by $\sim 5 \mathrm{MPa}$ than those in the parent grain.

\section{Discussion}

The present study reveals temporal (in stress) and spatial behaviour of the onset of plastic deformation in an embedded grain approximately $200 \mu \mathrm{m}$ below the surface of a tensile sample of magnesium alloy AZ31. Both basal slip and $\{10 \overline{1} 2\}<\overline{1} 011>$ twinning are shown to be the active deformation modes. Both of these systems are known to be readily activated in the present material [36] and both of these systems are favoured by the orientation of the grain selected for analysis. In the following, the sequence of plastic deformation is inferred and its spatial features are recapped. Then critical stresses are established and compared with the literature. The back stress in the twin is discussed after that and finally a few comments are made on some of the limitations and prospects of the present technique.

It is seen that plasticity begins in the studied crystal by the onset of basal slip. This is evidenced first by a distinct increase in the rate of grain rotation and, two load steps later, by the detection of distinct broadening of the Laue diffraction peaks. Twinning was then detected to initiate, first indicated by a drop in intensity of the parent reflections and then a 
load step later by the appearance of indexible twin related reflections. At its first appearance, the twin occupied the extent of its parent grain along at least one of its long axes, which is consistent with rapid twin propagation following nucleation. An increase in twin thickness of $\sim 20 \%$ was observed with an increase of applied stress. Inspection of the spreads seen in the Laue diffraction peaks revealed the likely presence of basal slip. The distribution of the peak spread over different locations in the grain revealed a concentration of plasticity at the twin tips. This reflects the initiation of additional slip activity to accommodate the twinning shear. The resolved twin shear stresses adjacent to and within the twin confirm previous findings [17] and theoretical considerations [9] that show that twinning relaxes the local stresses.

The first sign of basal slip is detected at applied stresses between 43 and $46 \mathrm{MPa}$. The corresponding resolved shear stress on the most heavily stressed system is $18 \mathrm{MPa}$. Due to the plateau in the stress curve, a similar value is obtained even if a later onset point is considered. The noise evident in the mean resolved shear stresses within the elastic regime enable us to give this value with an error of $+/-3 \mathrm{MPa}$. The corresponding value for twin formation (at an applied load between 54 and $58 \mathrm{MPa}$ ) falls in the range of 21 to $25 \mathrm{MPa}$ so we can give it as $23.5 \mathrm{MPa}+/-5 \mathrm{MPa}$. This value compares well with that corresponding to the onset of twinning, also in an embedded grain of approximately similar size and in a similar alloy, by Aydiner et al. [17] of between 22 and $42 \mathrm{MPa}$.

The ratio of the critical resolved shear stress for twinning to basal slip, which is $\sim 1.3$ is somewhat lower than what is normally inferred to be the case [5]. Values in the range of 2-7 have been reported for the present material. Most of these values, however, have been obtained by fitting crystal plasticity simulations to different orientation classes. This approach is particularly sensitive to twinning in the grains most favourably oriented for it. It may well be that in cases such as the present that basal slip activity impacts upon twin nucleation, thus leading to lower stresses for nucleation. Such is suggested by recent diffraction work [37] although the effect was not evident in electron back scatter diffraction analysis by Barnett et al. [38] by samples tested in compression. Further work is needed to clarify the strength and prevalence of this effect.

The occurrence of a back stress in a newly formed twin is a natural occurrence of the localization of the twinning shear within the twinned volume and the constraint imposed by the surrounding matrix, as is evident from the Eshelby inclusion formalism [9]. The shear stress required to move twinning dislocations over the interface of a growing twin must be 
matched by the interior stresses of the twin. Thus, we conclude that the critical resolved shear stress for twin growth in the present case is around 5 to $7 \mathrm{MPa}$, in accordance with values obtained in an earlier study [39].

The present technique has proven to be well suited to the present problem for onset of slip and deformation twinning. The improved temporal response provides a rapid mapping tool to extract the grain/twin morphology and underlying evolution of orientation and strain with fine imposed loading increments. For the time being, fast data acquisition limits analysis to the deviatoric strain components due to the limited energy response of X-ray area detector technology. However, with emerging energy resolving detectors access to the full strain tensor can be realised in the future, See for example: S. Send et al [40]. This energy resolution will also ensure higher levels of imposed load can be examined since crystallographic indexation will be more straight-forward.

Due to the relatively fine spatial nature of twin nucleation, the lower limit of twin detection appears to be a thickness in excess of about $5 \mu \mathrm{m}$. Below this length scale, data collection rates may have to be slowed by an order of magnitude to achieve the necessary counting statistics required for crystallographic indexation of weak/small diffracting twin domains. 


\section{Conclusions}

A new rapid mapping protocol was developed to provide an unprecedented combination of time and spatial resolution. We have used this approach to provide unique insight into yielding by slip and twinning for a magnesium grain embedded $\sim 200 \mu \mathrm{m}$ below the sample surface. Resolved shear stresses to an accuracy of about +/- $8 \mathrm{MPa}$ was achieved. Yielding was detected using intensity change, orientation change, mosaic spread and stress relaxation.

Yielding was observed to occur first by basal slip at a critical resolved shear stress of $18 \pm 3$ $\mathrm{MPa}$ and then by twinning at a resolved stress of $23.5 \pm 5 \mathrm{MPa}$. Basal slip occurred prior to twinning and in a concentrated manner to relax the stresses at the twin tips and interior immediately following twin formation. Twin growth was seen to occur at resolved stresses of between 5 and $7 \mathrm{MPa}$. 


\section{References}

[1] Christian J. W, Mahajan S. Progress in Materials Science 1995;39:1.

[2] Barnett M. R, Nave M. D, Ghaderi A. Acta Materialia 2012;60:1433.

[3] Muránsky O, Barnett MR, Carr DG, Vogel SC, Oliver EC. Acta Materialia 2010;58:1503.

[4] Muránsky O, Barnett MR, Luzin V, Vogel S. Materials Science and Engineering: A 2010;527:1383.

[5] Muránsky O, Carr D. G, Sittner P, Oliver E. C. International Journal of Plasticity 2009;25:1107.

[6] Agnew S. R, Brown D. W, Vogel S. C, Holden T. M. Materials Science Forum 2002;404407:747.

[7] Clausen B, Tomé C. N, Brown D .W, Agnew S. R. Acta Materialia 2008;56:2456.

[8] Gharghouri M. A, Weatherly G. C, Embury J. D, Root J. Philosophical Magazine A 1999;79:1671.

[9] Lebensohn R .A, Tome C. N. Philosphical Magnazine A 1993;67:187.

[10] Agnew S. R, Mulay R. P, Polesaklii F. J, Calhoun C .A, Bhattacharyya J. J, Clausen B. Acta Materialia 2013;61:3769.

[11] Wang J, Beyerlein I. J, Tomé C. N. Scripta Materialia 2010;63:741.

[12] Serra A, Bacon D. J, Pond R. C. Acta Materialia 1999;47:1425.

[13] Serra A, Bacon D. J. Philosophical Magazine A 1996;73:333.

[14] Yoo M. H. Transactions of the Metallurgical Society of AIME 1969;245:2051.

[15] Bashmakov V. I, Bosin M. E, Lavrentev F. F. Physica Status Solidi (A) Applied Research 1974;22:305.

[16] Bashmakov V. I, Bosin M. E, Lavrentev F. F. Problemy Prochnosti 1972;12:74.

[17] Aydiner C. C, Bernier J. V, Clausen B, Lienert U, Tome C. N, Brown D. W. Physical Review B Condensed Matter and Materials Physics 2009;80:024113.

[18] Balogh L, Niezgoda S. R, Kanjarla A. K, Brown D. W, Clausen B, Liu W, Tomé CN. Acta Materialia 2013;61:3612.

[19] Larson B. C, Yang, W., Ice, G. E.,Budai, J. D.,Tischler, J. Z. Nature 2002;415:887.

[20] Kunz M, Tamura N, Chen K, MacDowell A. A, Celestre R. S, Church M. M, Fakra S, Domning E. E, Glossinger J. M, Kirschman J. L, Morrison G. Y, Plate D. W, Smith B. V, Warwick T, Yashchuk V. V, Padmore H. A, Ustundag E. Review of Scientific Instruments 2009;80:035108.

[21] Lynch P. A, Parry D, Liang D, Kirkham R, Davey P, Stevenson A. W, Bettles C. J, Gibson M. A, Tomus D. Journal of Applied Crystallography 2011;44:610.

[22] Tamura N. XMAS: a versatile tool for analyzing synchrotron $x$-ray microdiffraction data. In: Barabash R, Ice, G. , editor. Microdiffraction Analysis of Local and Near Surface Hierarchical Organization of Defects. London: Imperial College Press, London, 2013. Poshadel A, Dawson P, Johnson G. Journal of Synchrotron Radiation 2012;19:237. Bourgeois D, Nurizzo D, Kahn R, Cambillau C. Journal of Applied Crystallography 1998;31:22. Larson B. C, Tamura, N., Chung, J.-S., Ice, G. E., Budai, J. D., Tischler, J. Z., Yang, W., Weiland, H., Lowe, W. P., editor 3-D Measurement of deformation microstructure in $\mathrm{Al}(0.2 \%) \mathrm{Mg}$ using submicron resolution white x-ray microbeams: Materials Research Society, 1999.

[27]

[29] Schmid E, Boas W. Plasticity of crystals : with special reference to metals. London: Chapman and Hall, 1935.

[30] Kelley E. W, Hosford J, W. F. Transactions of the Metallurgical Society of AIME 1968;242:5.

[31] Agnew S. R. Deformation mechanisms of magnesium alloys. In: Bettles CJ, Barnett M, editors. Advances in wrought magnesium alloys : fundamentals of processing, properties and applications. Cambridge, England :: Woodhead, 2012. p.63. 
[32] Barabash R, Ice G. E, Larson B. C, Pharr G. M, Chung K. S, Yang W. Applied Physics Letters 2001;79:749.

[33] Bieler T. R, Crimp, M. A., Yang, Y., Wang, L., Eisenlohr, P., Mason, D. E., Liu, W., Ice, G. E. Journal of The Minerals, Metals and Materials Society 2009;61:45.

[34] Basinski Z. S, Szczerba M. S, Niewezas M, Embury J. D, Basinski S. J. La Revue de Metallurgie 1997:1037.

[35] Agnew S. R, Brown D. W, Tomé C. N. Acta Materialia 2006;54:4841.

[36] Roberts C. S. The deformation of Magnesium. New York: Wiley, 1960.

[37] Raju K. S, Lynch, P. A., Barnett, M. R. Mat. Sci. For. 2014;773-774:104.

[38] Barnett M. R, Ghaderi A, Robson J. D. Metallurgical and Materials Transactions A: Physical Metallurgy and Materials Science 2013;45A:3213.

[39] Barnett M, Setty M, Siska F. Metallurgical and Materials Transactions A: Physical Metallurgy and Materials Science 2013;44A:2962.

[40] Send, S., Leitenberger, A. A., Weiss, M. S., Hartmann, R., Struder, L., Pietsch, U. J. Applied Crystallography 2012;45:517. 


\section{Acknowledgements}

We wish to thank J. Vella for tensile sample preparation and A. Sullivan for electron microscopy analysis and preparation of fiducial markers. The Advanced Light Source is supported by the Director, Office of Science, Office of Basic Energy Sciences, Materials Science Division, of the US Department of Energy under contract No. DE-AC02-

05CH11231 at Lawrence Berkeley National Laboratory. P. A. L would also like to acknowledge travel funding provided by the International Synchrotron Access Program (ISAP) managed by the Australian Synchrotron and funded by the Australian Government. 
Figure 1: Summary of the single shot tensile loading experiment a) Schematic illustration of the tensile loading system instrumentation, b) detailed view of the voxel grain data at a single XY point - the coloured grains lie beneath each other, starting with the red grain at the sample surface and extending in towards the sample centre; they each contribute to the measured Laue pattern but their diffraction centres differ (see image in a for definition of abbreviations).

Figure 2. Representations of the grain selected for analysis and the twin that appeared within it: a) basal pole figure with circled pole corresponding to selected parent grain, b) 3D representation showing voxel Euler theta data $(\phi 1: 123.74, \theta: 79.21, \phi 2:-154)$ for the parent grain (created using detector triangulation prior to the test) and c) twin location and habit plane.. In b) and c) the shaded pixelated plane on the left represents the data projected onto the location of the sample surface.

Figure 3. a. Experimental results showing the trend in the deviatoric shear strain terms with displacement of the point of origin from the calibrated 'zero' sample surface reference position. b. Comparative study of grain depth recovery across a single grain showing the results based on detector triangulation and single-shot methodology according to the measured deviatoric shear strain offset.

Figure 4. Grain average integrated intensity of the parent (21) 0$)$ and twin $(\overline{2} 114)$ reflections. 
Figure 5: Greyscale representation of the measured (21후) integrated intensity across the parent projected on the scanning XY reference frame at a) $54 \mathrm{MPa}$, b) $64 \mathrm{MPa}$ and c) $68 \mathrm{MPa}$. RGB colour representation of the measured $(\overline{2} 114)$ integrated intensity for the twin is also shown. The crystallographic habit plane trace is shown by a dashed line in b).

Figure 6. a) Misorientation evolution of a single diffraction point within the parent grain during loading. b) Evolution of the grain average mosaic spread for the parent and twin with appplied load.

Figure 7: Mosaic spread in the Laue $(21 \overline{3} 0)$ parent $(a, b)$ and $(\overline{2} 114)$ twin (c) reflections in terms of the FWHM $\left({ }^{\circ}\right)$. Twin location indicated by dashed line in a) and b). Evidence for increased 'accommodating' slip activity in the regions of the twin tips can be seen in b).

Figure 8. Experimental and simulated (red) Laue reflections for the parent (left) and twin (right) based on (0002)[11̄̄0] basal slip model. 
Figure 9. a) Spatial distribution and b) histogram of the resolved shear stresses on the most heavily stresses basal system across the grain of interest at an applied load of $38 \mathrm{MPa}$, which is in the elastic region. The blue curve in b) is a normal distribution with a standard deviation of 3.2 MPa.

Figure 10. a) Spatial distribution and b) histogram of the resolved shear stresses on the active twinning system across the grain of interest at an applied load of $64 \mathrm{MPa}$. The blue curve in b) is a normal distribution with a standard deviation of $3.4 \mathrm{MPa}$.

Figure 11. Values of the resolved shear stress on the a) most heavily stressed basal slip system and b) active twinning system, averaged over the parent grain and the twin. Minor relaxations of the basal stresses are seen in the twin but significant relaxation of the twin stresses are evident. The blue lines refer to elastic predictions assuming iso-stress conditions over the sample. 

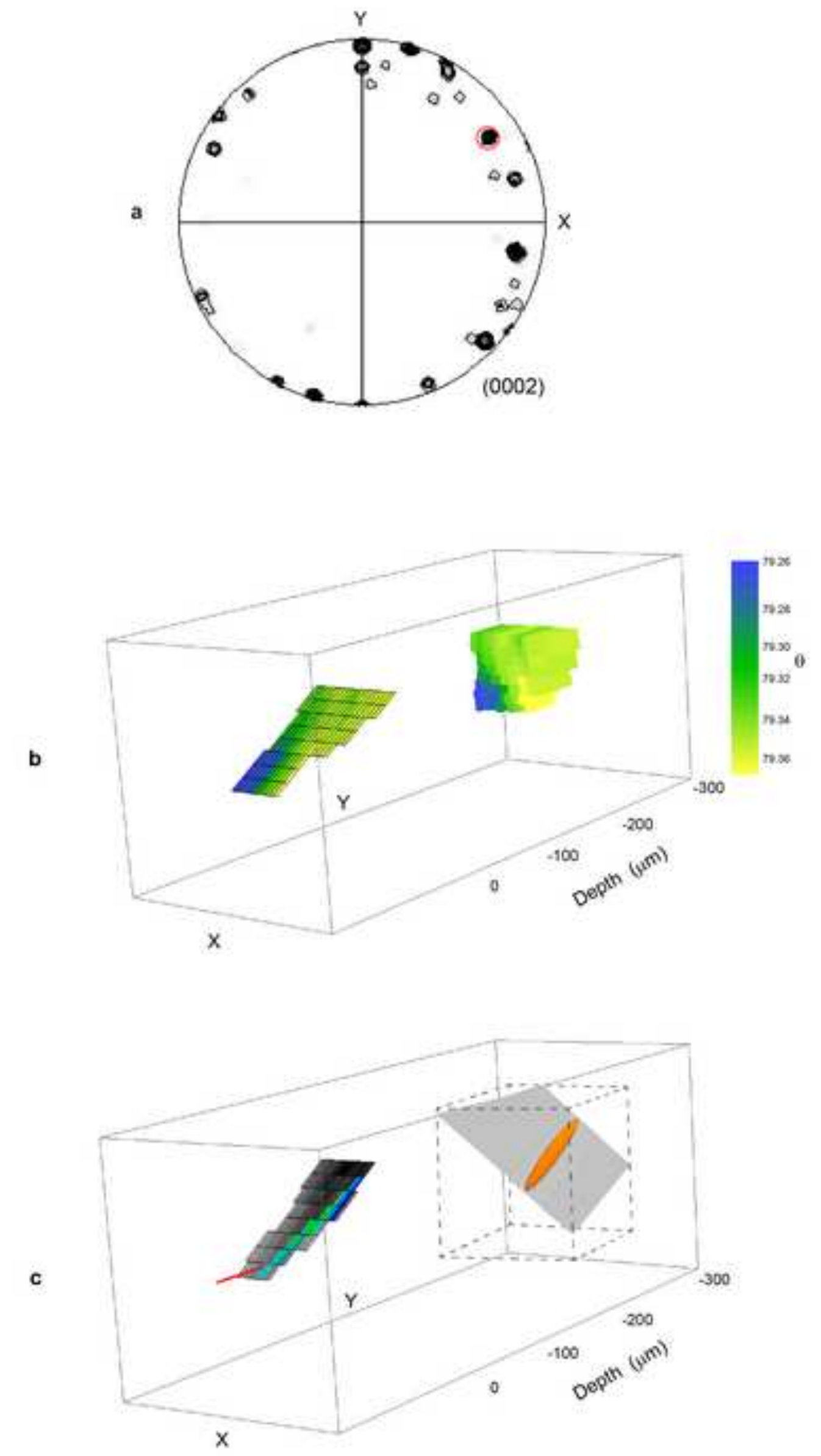

Figure(s)2

x

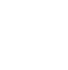

Figure(s)2

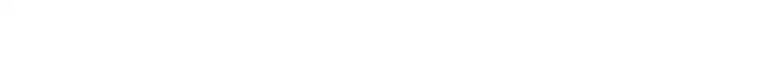

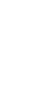

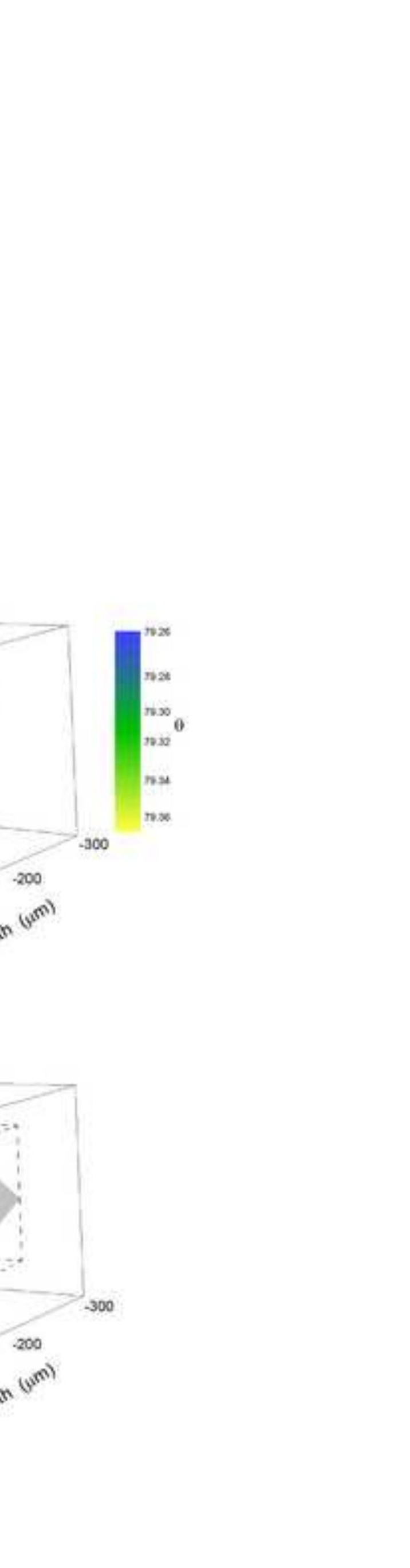

( 
a

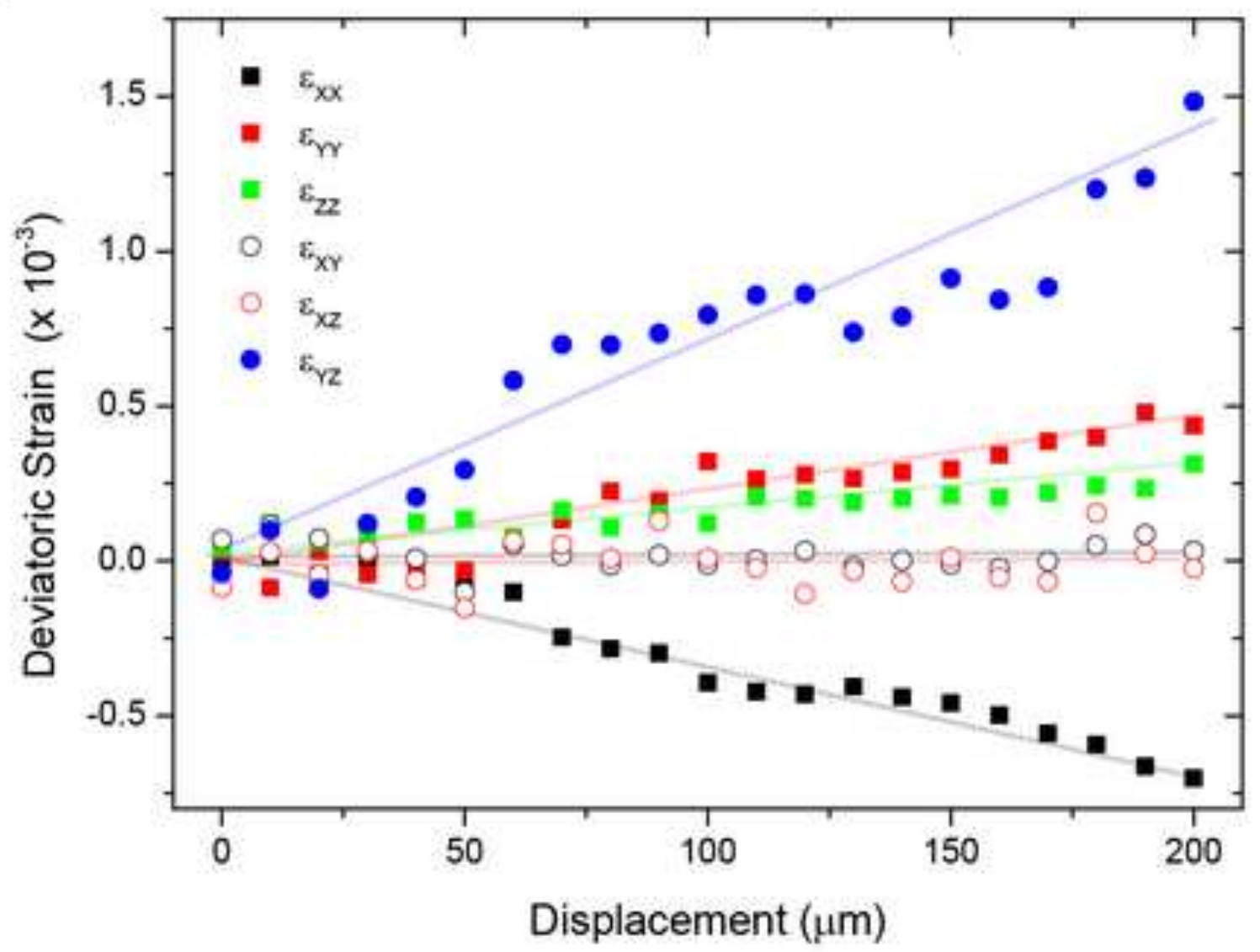

b

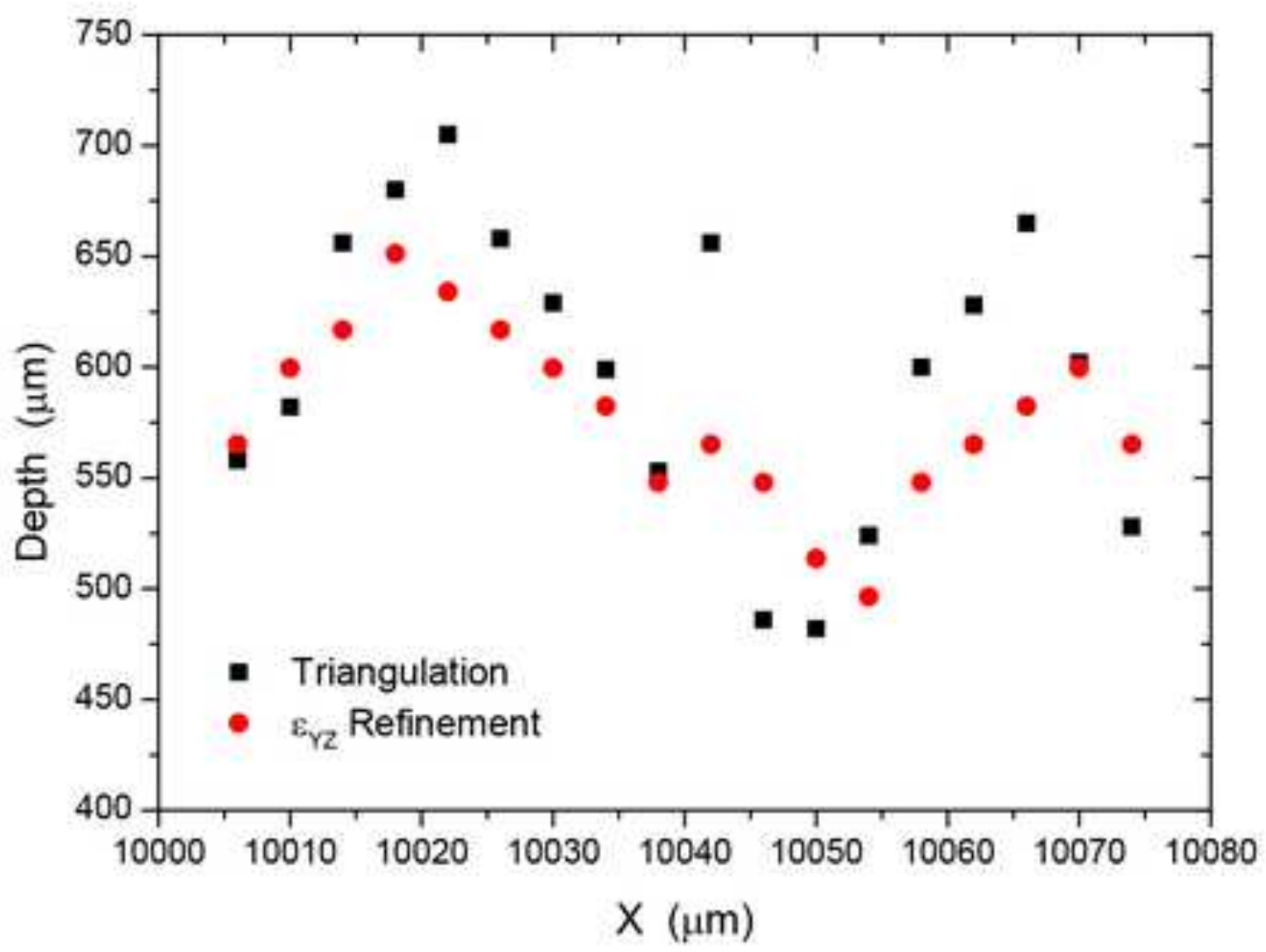




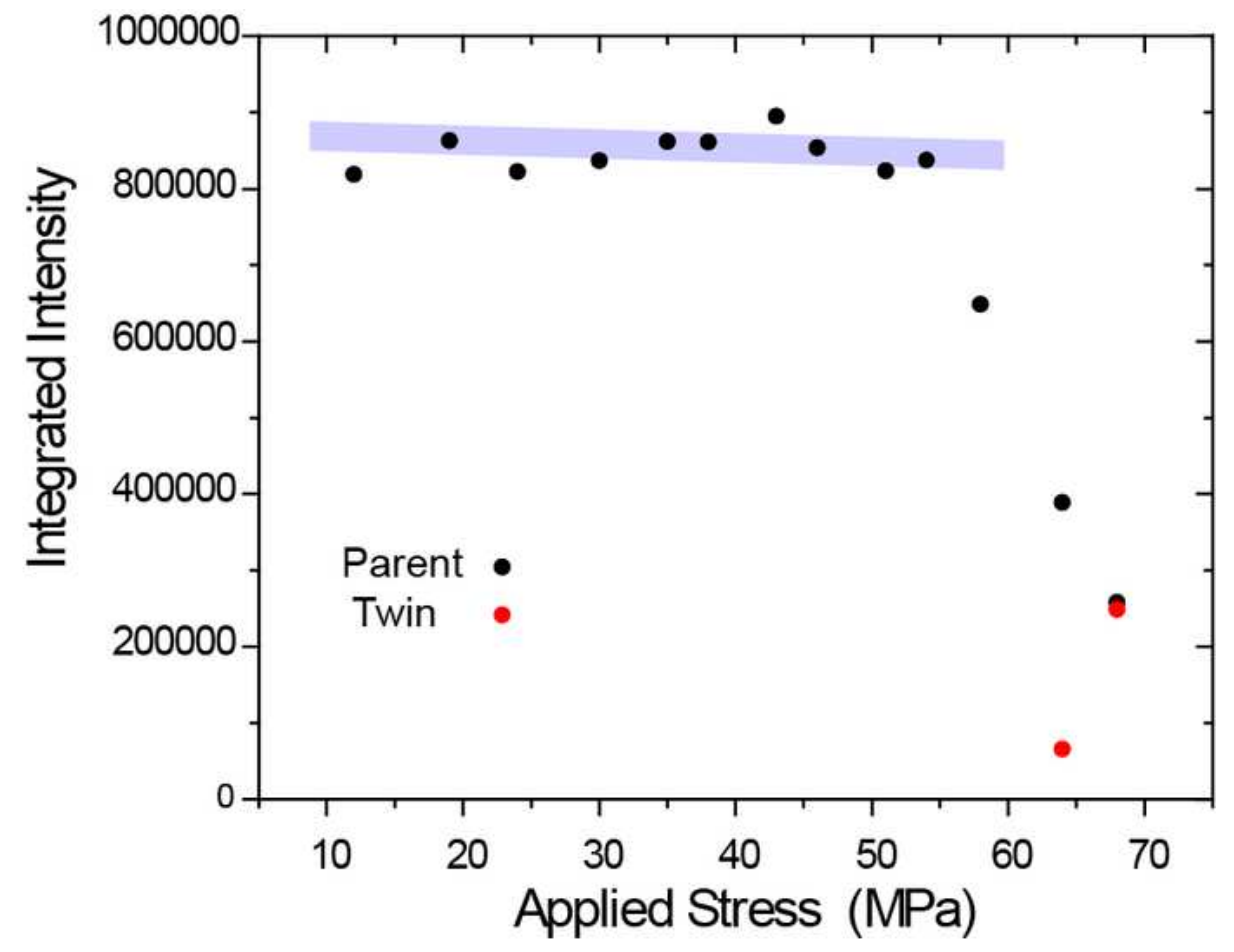




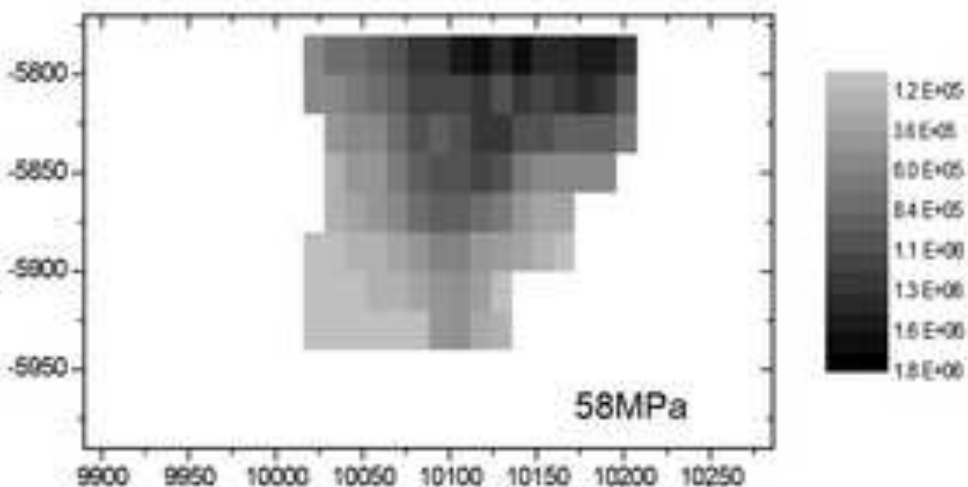

b
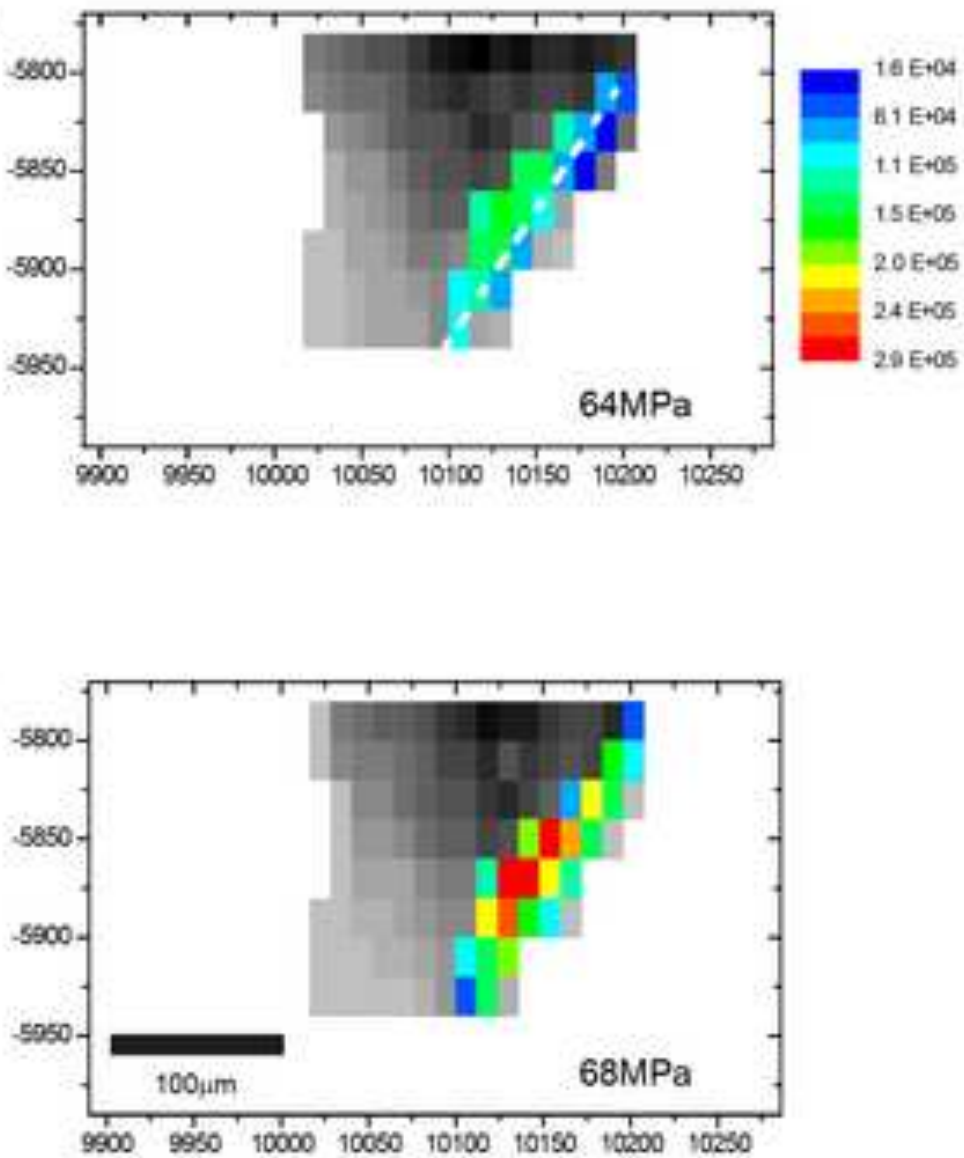
a
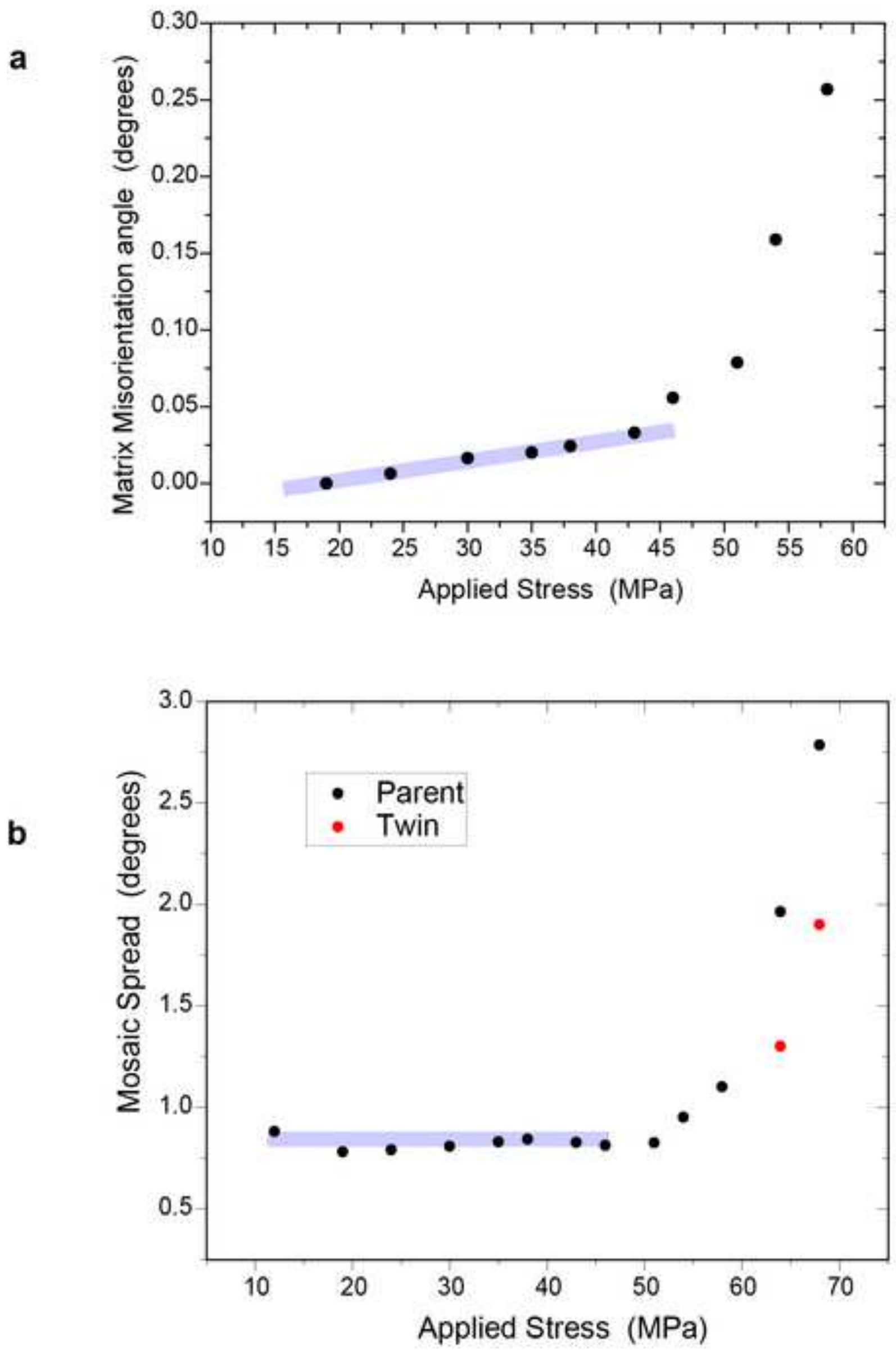
Figure(s)7

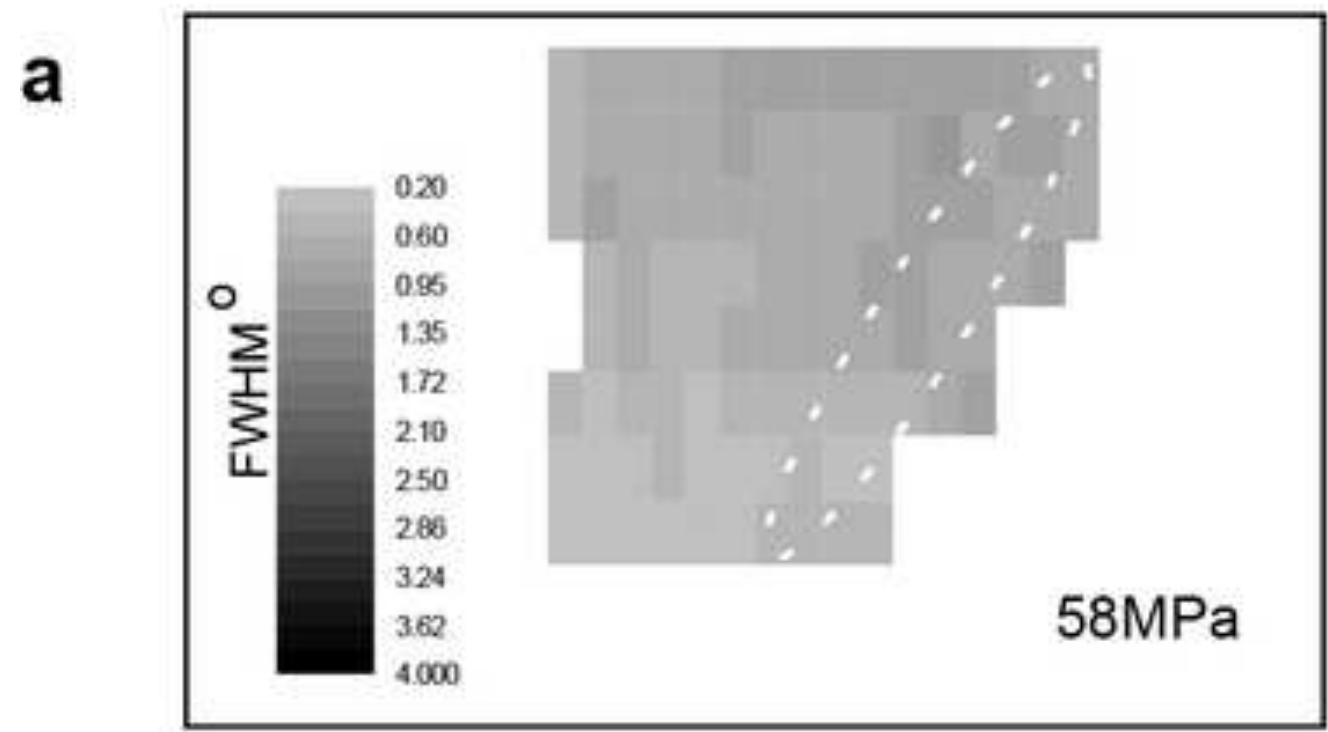

b

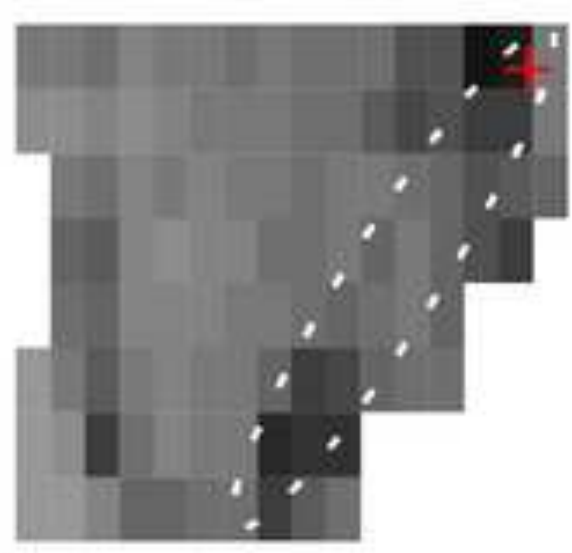

$100 \mu \mathrm{m}$

$64 \mathrm{MPa}$

C

$68 \mathrm{MPa}$
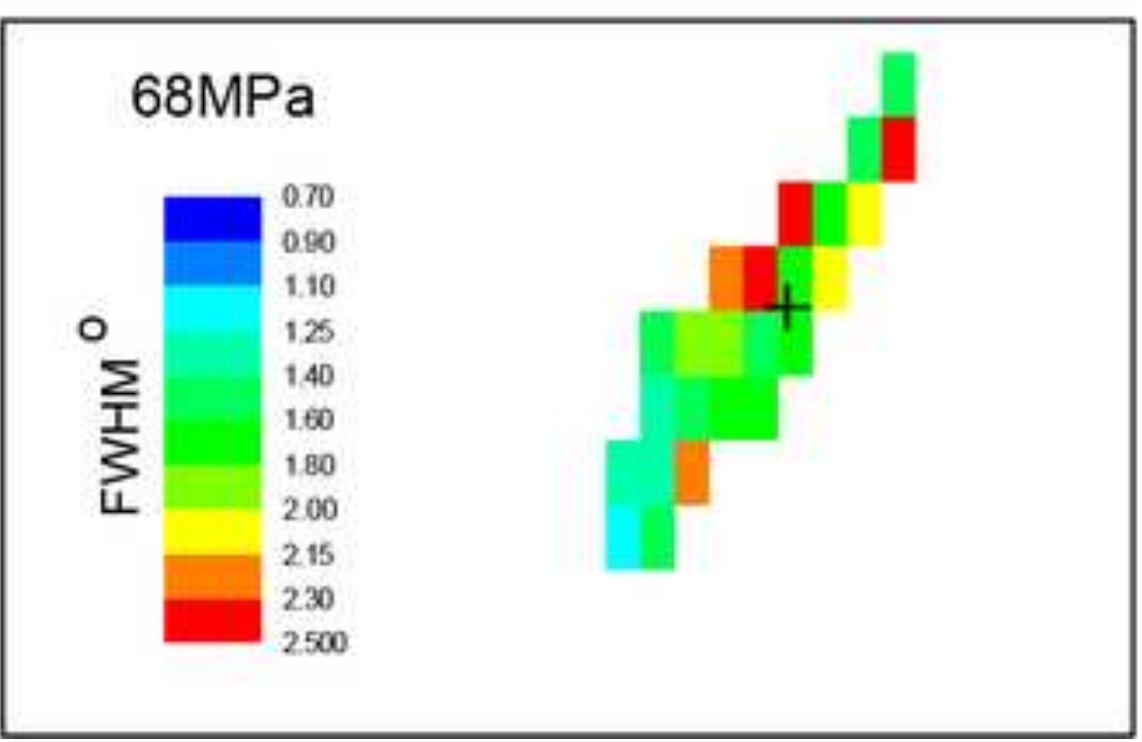

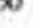

$58 \mathrm{MPa}$ 
Figure(s)8
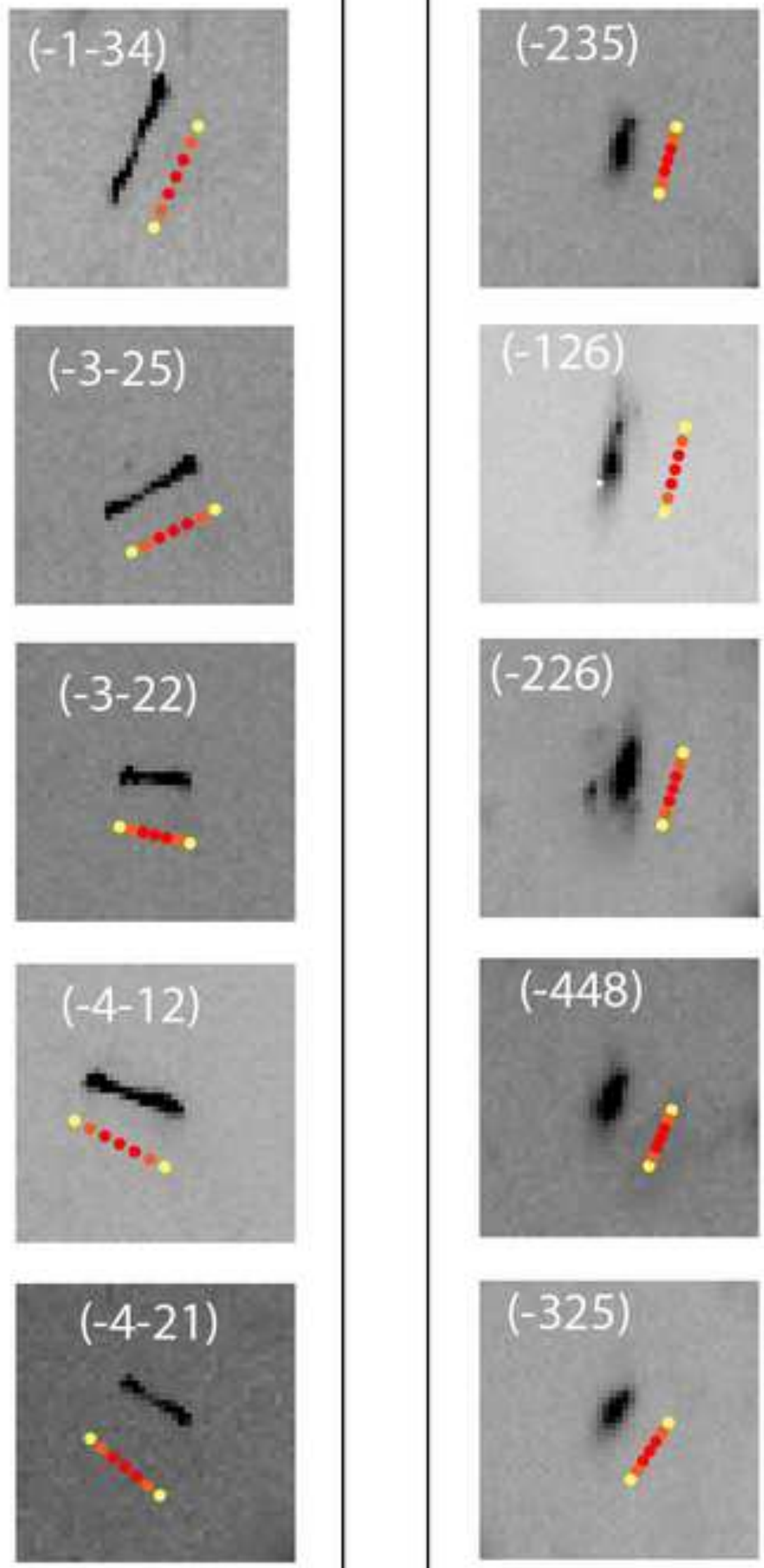

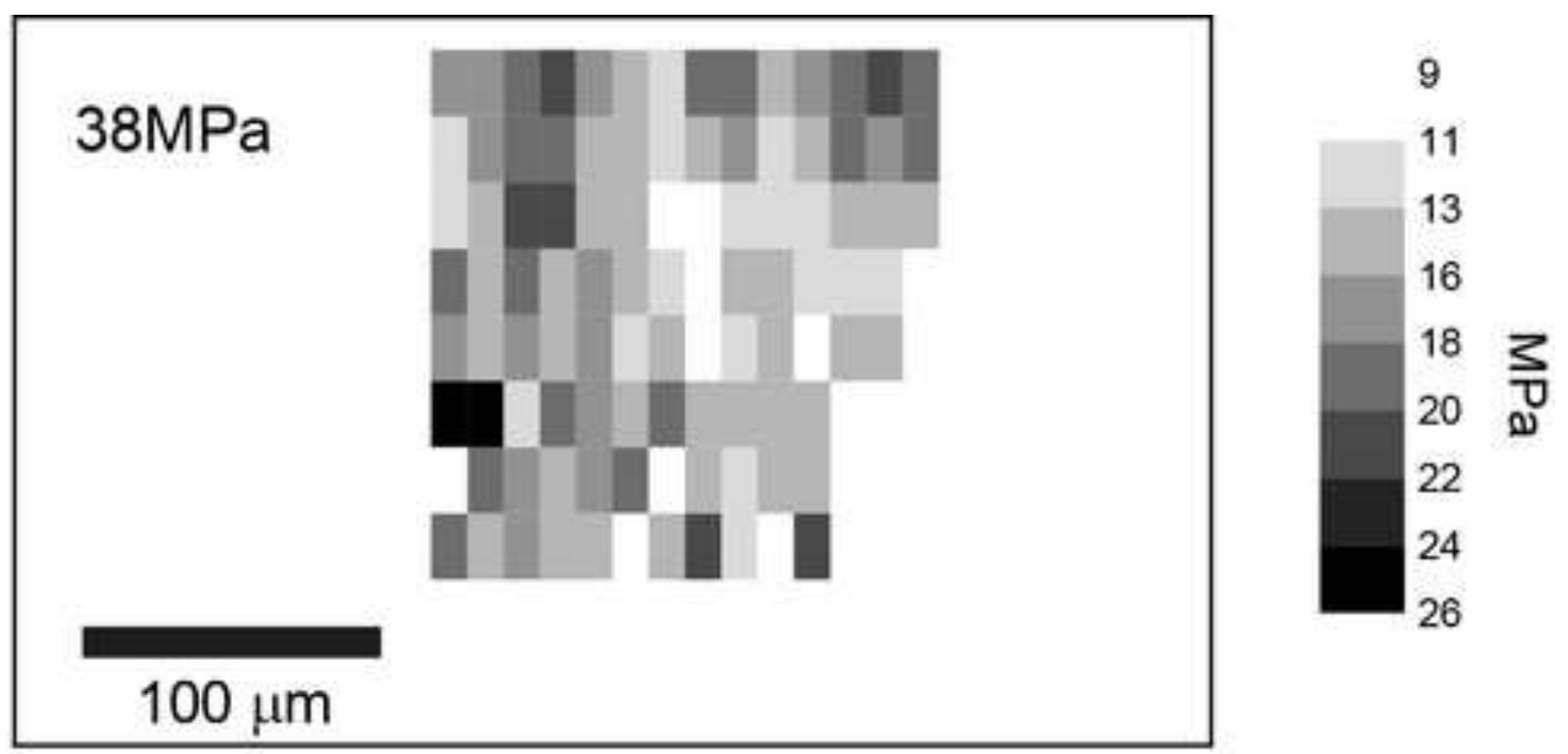

a

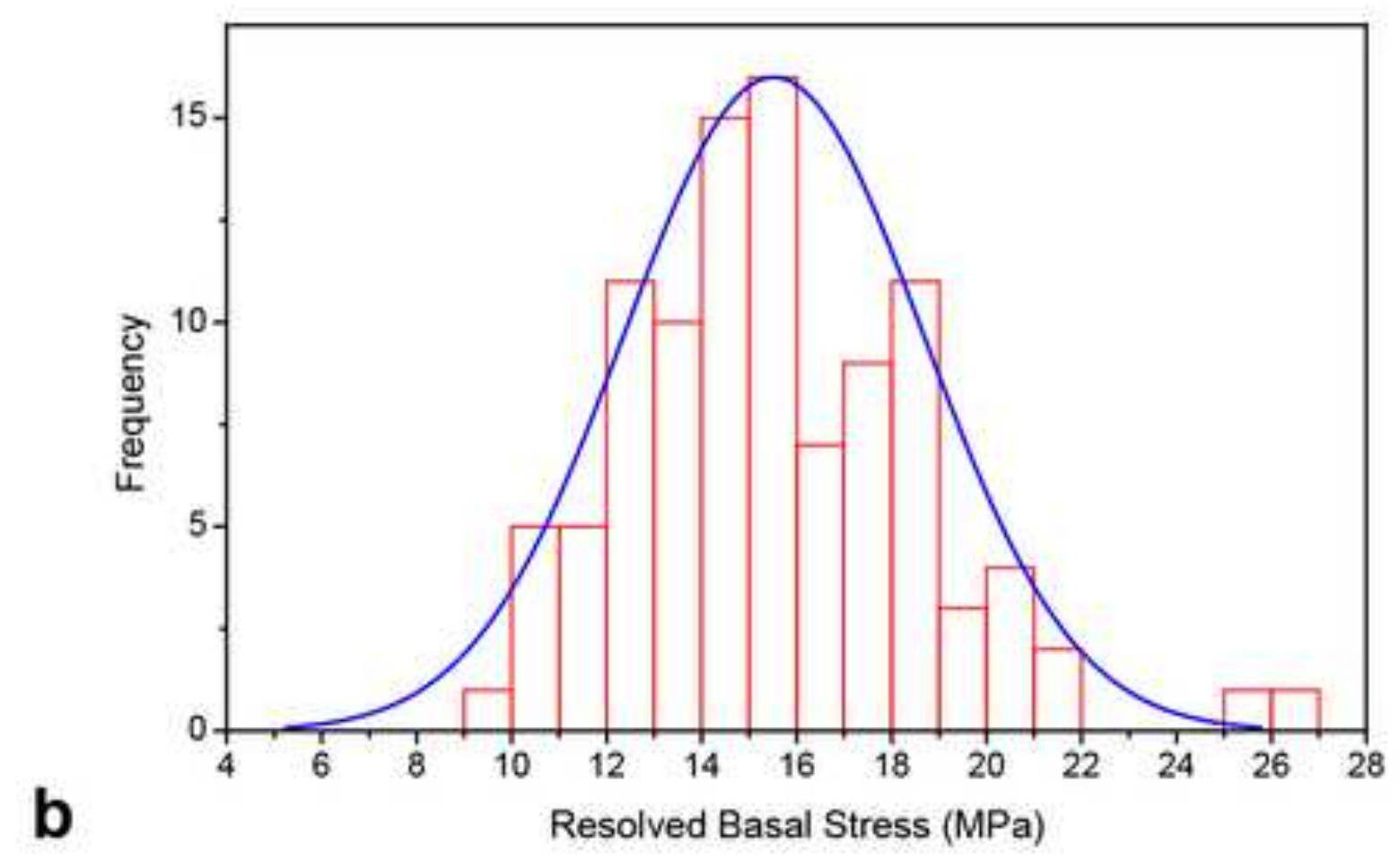


Figure(s)10
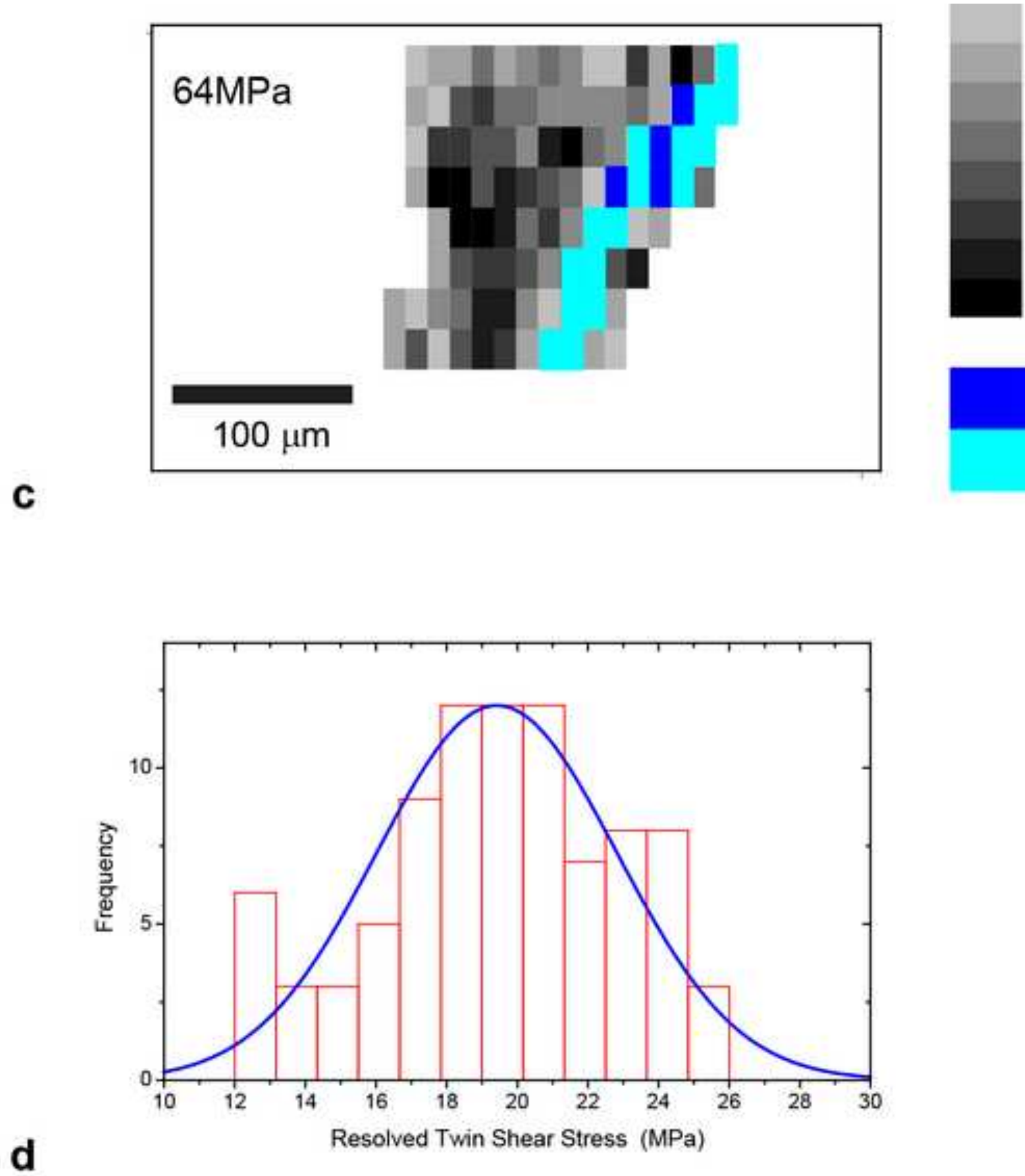

d 

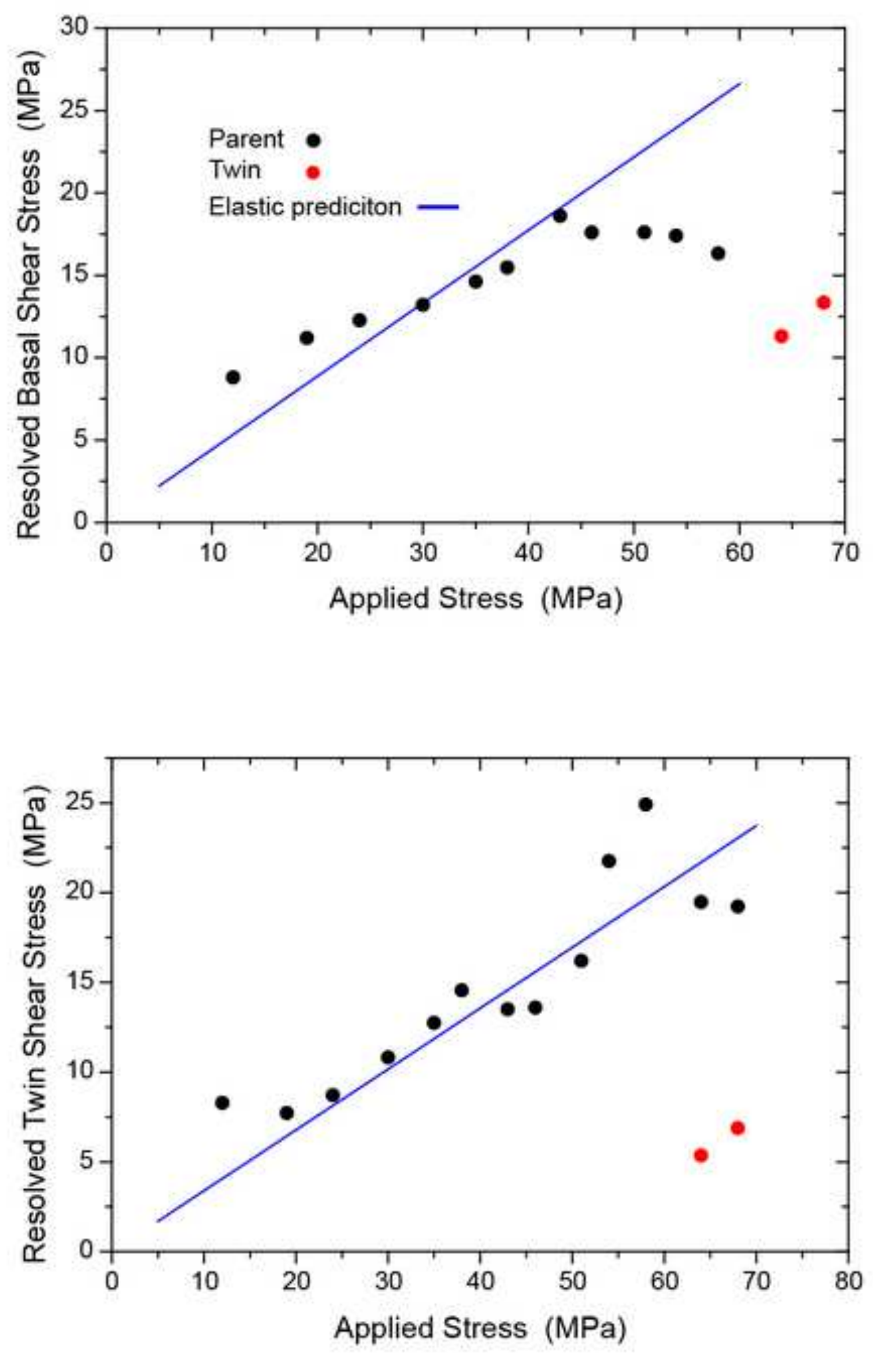

b

a 